\title{
Electromagnetic-Deformation Response of the Crust
}

\author{
Vladimir Uvarov,* \\ Institute of Cosmophysical Research and Radio Wave Propagation (IKIR) FEB RAS, 684034, v. \\ Paratunka, Kamchatka, Russia
}

\begin{abstract}
The analysis of mechanic-electromagnetic effects of the Earth's crust made it possible to distinguish the relation of rock induced polarization with its deformation. On the basis of these relations, their generalized description is made. It is shown that, although the Earth's crust is an energy-saturated structure in a critical state, but owing to electromagnetic properties, it is not a medium in which electromagnetic oscillations can be generated in the low-frequency region. At the same time, the Earth's crust is favourable environment for the occurrences of seismo-acoustic oscillations. It is shown that the mechanisms of mechanicelectromagnetic transformations of the crust generate electromagnetic waves accompanying seismic-acoustic disturbances. Examples of these waves manifestations in electric currents and in electromagnetic radiation are given.
\end{abstract}

Key words: seismo-electromagnetism, electrodynamics of the lithosphere, electromagnetic manifestation of earthquakes, electromagnetic waves accompanying deformation, Earth's natural electromagnetic field, electromagnetic radiation of the lithosphere

\section{INTRODUCTION}

Electromagnetic nature of solid body formation is the basis of electromagnetic manifestation of lithosphere dynamics.

Deformation of solid bodies including rocks is accompanied by electromagnetic radiation determined by mechanic-electromagnetic mechanisms. Some of these mechanisms are known quite well and have practical application, for example, the piezoelectric and piezomangeitc effects. All the mechanisms have physical models of various levels of detail and theoretical underpinning [1 - 6].

Some of them are applied quite often to interpret observable phenomena, field and laboratory experiments.

\footnotetext{
* Corresponding author: uvarovvnng@yandex.ru
} 


\section{CRUST AND GENERATION}

Electromagnetic radiation can be divided into two main types: equilibrium or thermal radiation, which remains in thermodynamic equilibrium with a matter, and a nonequilibrium (non thermal) radiation occurring in non-equilibrium systems during energy flux propagation and transformation in them.

The equilibrium radiation spectrum follows the Plank's law

$$
u(\omega, T)=\frac{\omega^{2}}{\pi^{2} c^{3}} \cdot \frac{h \omega}{\exp \left(\frac{h \omega}{k T}\right)-1}
$$

and is determined only by temperature $\mathrm{T}$ and frequency $f=2 \pi \omega$. .

The non-equilibrium spectrum is typical for non-equilibrium systems and is determined by the character of energy transformation processes occurring in the system. Examples of nonequilibrium radiation spectra are the spectra of flicker noise, synchrotron radiation, super luminescence. When inverse relations exist in a system, characteristic generation bands are formed just like it occurs in lasers, $L C / R C$ and Van der Pol generators.

As long as the Earth's crust is a non-equilibrium system in critical state, when investigating electromagnetic manifestations of the crust dynamics, we should clarify the possibilities of the Earth's crust as electromagnetic oscillation generator.

To generate oscillations, a structure must contain the following components:

- potential energy capacity used during the transformation into oscillation kinetic energy;

- converter of potential energy into oscillation kinetic energy;

- process control system for the potential energy transformation into oscullation energy;

- positive feedback circuit to control the transformation system.

Two regimes of occurring oscillations are possible - stable and unstable ones.

For an oscillating process to be stable, it is necessary to fulfil two conditions.

1. Energy consumption for one oscillation should be small in comparison to the accumulator capacity:

$$
\frac{\dot{E}_{a}}{E_{a} \omega}=\frac{1}{Q} \ll 1 .
$$

$Q \quad$ is the $\mathrm{Q}$-value. It means that the system $\mathrm{Q}$-value should be significantly more than a unit.

2. The accumulator self-discharge time should be large compared to the oscillation period $\tau \omega \gg 1$.

For typical values of the Earth's crust resistivity $\left(1<\rho<10^{4}\right) \quad[\Omega m]$ and dielectric permeability ( $5<\varepsilon<20$ ) the characteristic time of charge relaxation in the crust $\tau=\rho \varepsilon$ is within the range of $10^{-7}<\tau<10^{-2} \mathrm{~s}$ depending on environment parameters. 
In other words, the rock accumulating properties admit the possibility of generation occurrence in a rock depending on its parameters in the range of more than $10^{7}<\omega<10^{2} \quad \mathrm{~Hz}$.

However, the absence of positive feedback mechanism rules out completely the possibility of stable generation mode in the crust rocks.

Roughly speaking, the unstable electromagnetic oscillation regime is not associated with system generative properties. For unstable oscillations to be formed, the presence of electromagnetic-deformation response is enough which is determined by the rock mechanic-electromagnetic transformation mechanism (METM).

There is another situation for acoustic oscillations. A rock is a conglomerate of rockforming minerals in different phase states, the crystal matrix of which is capable of accumulating the mechanic stress potential energy. The characteristic time of rock being at stress state depends much on temperature.

The positive parametric inverse relation in rock medium (deformation resistance decrease during deformation rate increase characteristic for dry friction of solid bodies and thixotropic fluids) is confirmed both by earthquake occurrences and laboratory experiments.

It is clear that crust rocks are a suitable medium for the relaxation generation of acoustic oscillations arising under the influence of tectonic stress and their electromagnetic manifestations.

\section{Types of METM}

Electromagnetic signals of lithospheric origin (EM) accompanying earthquakes are associated with tectonic stress relaxation processes. Formation of these signals is determined by METM determined by the Earth's crust rock content and structure. Consideration of these mechanisms results in a number of consequences which inevitably affect the understanding of phenomenon physics and current approaches to the recording of signals of lithospheric origin. We analyze the known mechanisms of METM.

About $60 \%$ of the Earth's crust matter is silicon oxide which often occur in quartz form, has piezoeffect (electroelasticity, piezoelectric effect) applied in geophysical exploration [710]. Polarization $P$ change rate is proportional to deformation $\sigma$ rate

$$
|\dot{\boldsymbol{P}}| \sim|\dot{\boldsymbol{\sigma}}| \text {. }
$$

About $5 \%$ of the Earth's crust matter is iron having ferromagnetic properties. Ferromagnetic domain structure oriented by constant geomagnetic field suffers some distortions under the effect of seismo-acoustic disturbances or stress causing changes in magnetic induction and effective magnetic permeability $\mu \quad \mu[11,12]$ :

$$
|\dot{\boldsymbol{P}}| \sim \frac{\partial \mu}{\partial \sigma}|\dot{\boldsymbol{\sigma}}| \text {. }
$$

The Earth's crust rock is a conglomerate of minerals in different phases, they are: solid, fluid and gasous. Solid minerals are mainly represented by crystals, forming a matrix, in the pores and fractures of which are the fluids, i.e. fluid and gaseous minerals. Owing to the inequality of the work function at the boundary of different minerals, double electric layer is formed.

Motion of a fluid together with the charge causes current, proportional to the velocity of the fluid motion relatively a mineral matrix, that is an electrokinetic effect which results in 
transformation of acoustic oscillations into electric ones (Frankel-Biot waves) [13 - 16]. The effect was applied in geophysical exploration $[8,17]$.

Besides the effects mentioned above, a number of other effects of transformation of mechanic disturbances into electromagnetic waves are known:

- inertial effect is the ion analogue of Stewart-Tolman effect in a porous two-phase medium; it is associated with ion response time relatively moving fluid in rock pores $[2,18]$ :

$$
|\dot{\boldsymbol{P}}| \sim|\dot{\boldsymbol{\sigma}}|
$$

- induction effect during conducting medium motion in the magnetic field \$B $\$$ polarization is proportional to the product of magnetic field value by deformation rate $[2,18]$ :

$$
|\dot{\boldsymbol{P}}| \sim|\dot{\boldsymbol{\sigma}}| \cdot \boldsymbol{B}
$$

- deformation effect determined by conductivity change during rock dilatancy accompanied by fluid diffusion in pore channels and causing telluric current modulation $[2,18]$ :

$$
|\dot{\boldsymbol{P}}| \sim \frac{\partial \rho}{\partial \sigma} \cdot|\dot{\boldsymbol{\sigma}}|
$$

- effect determined by dislocation dynamics $[2,18,19]$ :

$$
|\dot{\boldsymbol{P}}| \sim|\dot{\boldsymbol{\sigma}}|
$$

- effect determined by the difference of charge carriers mobility during dislocation flow in rock crystals [20,21]:

$$
|\dot{\boldsymbol{P}}| \sim|\dot{\boldsymbol{\sigma}}| ;
$$

- effect caused by the change of dipole moments of microcracks with oppositely charged sides during rock cracking $[2,18,22]$ :

$$
|\dot{\boldsymbol{P}}| \sim|\dot{\boldsymbol{\sigma}}|
$$

- generation of a plasma discharge between the cracks sides [23]:

$$
|\dot{\boldsymbol{P}}| \sim|\dot{\boldsymbol{\sigma}}|
$$

- triboelectric effect determined by the difference of work function of contacting materials during separation:

$$
|\dot{\boldsymbol{P}}| \sim|\dot{\boldsymbol{\sigma}}|
$$

- generation of electromotive force from mobile crystal defects in mechanic stress gradient field [24]:

$$
|\dot{\boldsymbol{P}}| \sim|\dot{\boldsymbol{\sigma}}| \text {. }
$$


At last, we cannot but mention the global piezoeffect of the Earth's crust which is associated with the absence of inversion along the Earth's crust vertical axis due to the gravity field, rock pressure increase with depth and crust stratification during its formation. Based on Curie principle, this circumstance should cause global piezoelectric effect:

$$
|\dot{\boldsymbol{P}}| \sim|\dot{\boldsymbol{\sigma}}| \text {. }
$$

Evidently, this list does not exhaust all possible METM. We should note that the main effects (piezoelectric, piezomagnetic, electrokinetic and induction) are reversible in Hooke deformation region.

All these effects relate the deformationally induced polarization rate with deformation rate $\dot{\sigma}$. Taking into account all the previous ratios we can write the following ratio for the crust deformationally induced polarization:

$$
|\dot{\boldsymbol{P}}|=\left(A+B \frac{\partial \rho}{\partial \sigma}+C \frac{\partial \mu}{\partial \sigma}\right)|\dot{\boldsymbol{\sigma}}|
$$

Here $A, B, C$ are the constants determining the weight of each factor in the general balance.

These effects cause electromagnetic manifestation of lithosphere dynamics, in particular, electromagnetic waves accompanying seismo-acoustic disturbances $[4,17,25]$.

Investigation of these phenomena entailed introduction of the notion "seismoelectromanetism" as a field of natural medium radio physics.

\section{Radiation and Deformation}

Electromagnetic wave generation is a physical manifestation of medium electromagnetic structure nonstationarity. In its turn, the nonstationarity occurs during accelerated motion of electric charges of different polarity relatively each other under the effect of external forces. The processes described above take place during rock charge structure change during its deformation causing electromagnetic radiation of deformation origin.

In this case, the full output of the radiation is determined by Larmor relation [26]

$$
W=\frac{2}{3} \frac{(\ddot{\boldsymbol{P}})^{2}}{c^{3}} \text {. }
$$

Here $\quad \boldsymbol{P}=Q \cdot \boldsymbol{d}$ is the resultant dipole moment of a system, $\quad c$ is light velocity.

When considering a unit volume

$W$ is the intensity of radiation from elementary volume,

$\boldsymbol{P}$ is a degree of polarization in the deformation area,

$Q \quad$ is the charge density,

$\boldsymbol{d}$ is the effective base of equivalent dipole,

$\left({ }^{\prime}\right)$ is the second derivation with respect to time. 
For the sake of simplicity, we introduce an assumption that density deformation tensor is determined by the module of its main vector $\sigma$ and it is proportional to dipole moment base $\vec{d} \sim \sigma$. Substituting (13) into (14), we obtain

$$
\begin{aligned}
& W \sim \alpha^{2} \ddot{\boldsymbol{\sigma}}^{2}+2 \alpha \dot{\alpha} \sigma|\dot{\boldsymbol{\sigma}}|+\ddot{\alpha}^{2}|\boldsymbol{\sigma}|^{2}, \\
& \text { where } \quad \alpha=\left(A+B \frac{\partial \rho}{\partial \sigma}+C \frac{\partial \mu}{\partial \sigma}\right) .
\end{aligned}
$$

It is clear from this expression that electromagnetic radiation may be associated both with the character of polarization occurrence and with the character of deformation motion. It is reflected quite well in the character of correlation relations illustrated in Fig. 1.
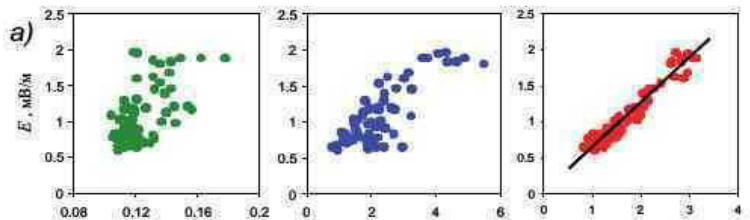

o, $m k m$

$\dot{\boldsymbol{o}}, \mathrm{mkm} / \mathrm{s}$
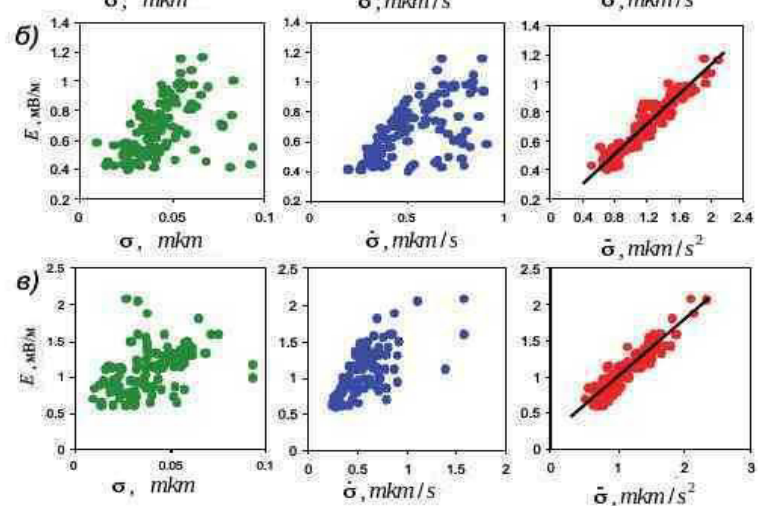

Fig1: Examples of correlation relations between the amplitude of electric pulse in the ground and seismic pulse parameters (amplitude, velocity, acceleration); data recorded at Tunkinskiy fracture zone during three sessions [27].

\section{Signal features}

It is clear from reasoning above and Fig. 1 that electromagnetic manifestation of crust deformation processes contains the information both on the crust mechanic motion parameters and on the crust electrophysical properties in the regions of deformation activity. In other words, transformation of a medium structure during deformation relaxation generates electromagnetic disturbance reflecting both the crust tectonic reconstruction dynamics and the character of its electromagnetic properties.

As long as the signals of atmospheric-lightning, ionospheric-magnetospheric and industrial origin, which in our case act as noise, are known quite well. It allows us to separate them from the signals of lithospheric origin especially in the areas of increased seismic activity and good electromagnetic ecology. 
The crust, as a complicated rheological medium, is characterized by a wide range of different mechanic motions from shock relaxation bursts accompanying brittle fractures to the flows typical for the water-saturated rocks. It is natural that this will be reflected in electromagnetic manifestations of relaxation processes. Analysis of electromagnetic radiation accompanying earthquakes showed quite a large set of wave manifestations recorded at Karymshina geophysical station of IKIR FEB RAS (Kamchatka), the most characteristic part of which is illustrated in Fig. 2.

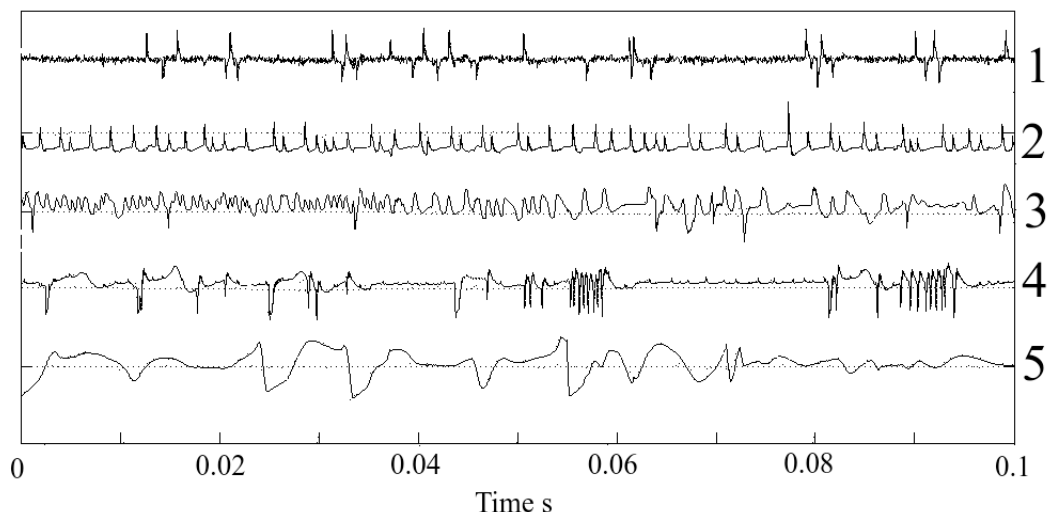

Fig 2. Typical forms of electromagnetic signals accompanying earthquakes. The horizontal axis is time in seconds.

Here the signals of type 1 (Fig. 2, short-time high-amplitude bursts), representing, to our opinion, an extreme event, may be associated with continuous brittle fracture each act of which is accompanied by a short-time pulse. The signals of type 5 should be referred to the opposite extreme event associated with a flow of rheological complicated medium in the conditions of variable tectonic stress. Intermediate cases $(2,3,4)$ may evidently be associated with relaxation processes with different degree of effect of these extreme cases.

However, the task of development of the methods for the crust geodynamic state diagnostics is presented unclearly in seismo-magnetism investigations [10, 11] though application of electromagnetic measurements of the field for geophysical exploration is arguable and requires close attention of researchers.

\section{Main results}

1. The analysis of mechanic-electromagnetic effects of the Earth's crust allowed us to detect the relation of induced polarization with its deformation.

2. Based on the obtained ratios for different deformation-polarization effects, we generalized their analytical description.

3. It has been shown that though the crust is an energy-saturated medium in a critical state, it is not the medium where electromagnetic oscillations are generated in the low-frequency range owing to the electromagnetic properties. At the same time, the Earth's crust is a favorable medium for generation of seismo-acoustic oscillations and they electromagnetic manifestations. 
4. It has been shown that the mechanisms of mechanic-electromagnetic transformation of the crust generate electromagnetic waves accompanying seismo-acoustic disturbances.

\section{Conclusions}

In spite of the principle clarity of the physical basis of the seismo-magnetizm, it is too early to discuss its practical application as a monitoring tool for geodynamics.

The main problems are determined by incomparability of the natural processes with their laboratory models in scale and conditions [8]. The sporadicalnes and unpredictability of lithosphere activation processes together with their inaccessibility for contact investigations makes it impossible to detect dominating mechanisms necessary for physical analysis.

The work was carried out by the means of the Common Use Center "North-Eastern Heliogeophysical Center" SKR 558279

\section{References}

1. M.A. Sadovsky, Electromagnetic Precursors of Earthquakes (M: Nauka, 1982)

2.V.Surkov, M.Hayakawa, Ultra and Extremely Low Frequency Electromagnetic Fields (Springer Japan 2014)

3. M. Krumbholz, Electromagnetic radiation as a tool to determine actual crustal stress application and limitation. (Dissertation zur Erlagung des Doktorgrades der Mathematisch-Naturwissetschaftlichen Fakultaten der Georg-August-Universitat zu Gottingen, Gottingen, 2010)

4. V.N. Uvarov, Geophysical Journal, 38, 6, (2016)

5. V.N.Uvarov, Geophysical Journal, 34, 6, (2012)

6. V.N.Uvarov, Bulletin of KRAESC. Phys.-Math. Science, 1, 12, ( 2016)

7. E.I. Parkhomenko, The phenomenon of electrification in rocks. (M: Science) 1(968)

8. B.S. Svetov , V.P. Gubatenko, Physics of the Earth. №10. (1999)

9. S.I. Skipochka, Mechanoelectric effects in rocks and their use in mining geophysics, (Dnepropetrovsk: National Mining Academy of Ukraine, 2002)

10. G.A. Sobolev, V.M. Demin Mechanoelectric phenomena in the Earth. (M: Science, 1980)

11. E. Villary, Ann. Phys. Chem. 126, 87, (1865)

12. D.C. Jiles, J.Phys. D: Appl. Phys. 28 (1995)

13. Ya. I. Frenkel, Izv. AN SSSR, 8, 4, (1944) 
14. M.A. Biot, J.Acoust.Soc.Am.m., 28, (1956a)

15. M.A. Biot, J.Acoust.Soc.Am.m., 28, (1956в)

16. S.R. Pride, Phys.Review, v 50, (1994)

17. B.S. Svetov, Geophysics, 1, 2000

18. A.V. Guglielmi, Physics of the Earth, №3, (2006)

19. R. Teisseyre, T.Ernst, Annals of geophysics, 45, 2, (2002)

20. V. Hadjicontis , C. Mavromatou, T. N. Antsygina, K. A. Chishko Phys.Rev.B 76, 2, (2007).

21. I.R. Shevtsova Physics of the Earth, 4, (1984)

22. A. Tzanis, F. Vallianatos, Seismo Electromagnetics Lithosphere-AtmosphereIonosphere Coupling, Eds. Hayakawa and Molchanov, (Tokyo:Terrapub, 2003)

23. M. I. Molotsky, Phys. Solid. Body, 25, I, (1983)

24. F. St-Laurent, J.S. Derr, F.T. Freund, Physics and chemistry of the earth. 31 (2006)

25. Y. Fujinawa and Y. Noda, Pure Appl. Geophys. (Springer Basel, 05.03 2015)

26. L. D. Landau, E. M. Lifshitz Theoretical physics. Field theory, (M:Nauka, 1988)

27. V.V. Adushkin, A.A.Spivak Physical fields in near-surface geophysics. (M: GEOS, 2014) 